\title{
Novel technique to measure mutual bulk fluid diffusion using NMR 1- D gradient
}

\author{
Son Dang ${ }^{*}$, Carl Sondergeld, and Chandra Rai \\ Mewbourne School of Petroleum and Geological Engineering, University of Oklahoma, USA
}

\begin{abstract}
Many modelling and theoretical studies have shown that diffusion can be a significant transport mechanism in low-permeability porous media. Understanding the process allows engineers to better predict reservoir performance during both primary production and enhanced recovery in unconventional reservoirs. Direct measurement of effective diffusion in tight rocks is difficult, due to small pore volumes and the lack of techniques to actually monitor the process. Conventional diffusion measurements generally require fluid sampling, which induces a pressure transient which changes the mass transfer mechanism. Previously, we introduced a novel technique to measure tortuosity in nano-porous media by simultaneously monitoring methane versus nitrogen concentrations at high pressure using transmission Infrared Spectroscopy (IR). To complete the estimation of effective diffusion, bulk fluid diffusion coefficient also needs to be measured. In this study, we demonstrate the usage of Nuclear Magnetic Resonance (NMR) 1-D imaging to examine the dynamic change of Hydrogen Index (HI) across the interface between two bulk fluids. The experiment was conducted between a crude oil sample and methane; fluid samples were pressurized within an NMR transparent $\mathrm{ZrO}_{2}$ pressure cell which operates at pressures up to 10,000 psi. The Hydrogen Index (HI) profile was continuously measured and recorded for 7 days. The results provided oil the swelling factor and the concentration profile as a function of both time and distance. These data then were fitted with MaxwellStefan equation to precisely back calculate the diffusion coefficient between oil and gas samples at high pressure. Accurate estimation of tortuosity and fluid diffusion is critical for the gas injection strategy in a shale formation. Greater tortuosity and smaller fluid diffusion rate lead to longer injection and production times

for desirable

economic

recovery.
\end{abstract}

\section{Introduction}

Recent studies, including simulation works (Li et al., 2018[1]), experimentation (Li et al., 2019[2]; Dang et al., 2019[3]), and production modelling (Cronin et al., 2018[4]), suggest that matrix diffusion is a major mass transport mechanism, along with advection. Advection of a flow in porous media is governed by fluid properties (such as viscosity, density, and compressibility) and matrix permeability. Diffusion is governed by fluid diffusivity (either free diffusivity or multi-component diffusivity) and porous media' tortuosity. The Sherwood number, commonly cited in surface science and catalyst studies, is used a dimensionless factor accounting to the relative contribution between diffusion and advection in overall mass transport (Coutelieris et al., 2002[5]). With high porosity media, such as conventional rocks, the impact of advection overpowers the role of diffusion. While in tight rocks, with matrix permeability in the order of nano-Darcy, the impact of diffusion is not negligible.

With the development of unconventional shale gas and oil, the need to reevaluate these transport mechanisms, especially in nano-porous media, is essential. This does not only apply for primary production, but also for Enhanced Oil Recovery (EOR) processes. The understanding of behaviors of light gas molecules diffusing into rock matrix with included reservoir fluids, is important to optimize the efficiency of gas injection. Tortuosity measurement in nano-porous media is limited. Recently, Fleury and Brosse, 2017[6] and Dang et al., 2018[7] proposed different techniques to estimate tight matrix's tortuosity. The results suggested for rock samples with porosity, less than $7 \%$, that tortuosity can vary from 4 to 25 , a considerable range. In contrast, tortuosity values for conventional rocks, with porosity greater than $10 \%$, are around 2 (Iversen and Jorgensen, 1993[8])

Besides matrix tortuosity, bulk fluid diffusivity also controls effective diffusion. The molecular diffusivity in the gas phase can be computed with Sigmund, 1976[9] correlation. In term of experimentation, this parameter can be estimated via several methods, including monitoring pressure profile while the oil phase contacts the gas phase inside a closed cell (Guo et al., 2009[10]). The drawback of this technique is that pressure is not maintained constant throughout the measurement. Another technique is monitoring the oil phase swelling while injecting gases at a constant pressure (Jamialahmadi et al., 2016[11]). The swelling data is acquired by tracking the change in elevation of oil-gas interface. However, with the pressure above first contact minimum miscibility pressure (MMP), the interface becomes so vague to defy precise monitoring. In this study, we propose a new method using NMR 1-D gradient to monitor the dynamic change between oil-gas interfaces, from which bulk fluid diffusivity can be determined. 


\section{Experimental setup and fluid samples}

For pressurization experiments, we used a Daedalus ${ }^{\circledR}$ cell, made of NMR transparent $\mathrm{ZrO}_{2}$; the cell can be operated up to $10,000 \mathrm{psi}$ internal pressure. The oil phase was injected into the pressure cell via a downstream port, while the gas phase would be later injected into the cell through the upstream port. The cell was then positioned inside the NMR spectrometer, in which the oil-gas interface would be aligned in the middle of the gradient scanning window. From its inlet, the cell was connected to a syringe pump system, which was used to compress gases from supply cylinders, then inject gasses into the test cell at a test pressure. The pressure was maintained constant throughout the diffusion process. Fig. 1 illustrates major components of the experimental setup.

NMR gradient profiles were acquired using Oxford 2 $\mathrm{MHz}$ GeoSpec ${ }^{\mathrm{TM}}$ spectrometers, and Green Imaging acquisition and processing software. The magnet temperature was set at $35^{\circ} \mathrm{C}$ throughout the experiments. The gradient scanning window was set at $7 \mathrm{~cm}$, using DNK sequence. A new scan was repeated every 1 hour. Dynamic change of hydrogen index (HI) profiles across the oil-gas interface during the diffusion process, allows us to extract bulk diffusion parameters. In this study, the fluid samples included a dead oil from Meramec formation, and the injection gas is methane. Fig. 2 represents a HI profile at the beginning of a 7-days experiment with methane injection pressure of $6000 \mathrm{psi}$, which is above minimum miscibility pressure (MMP).

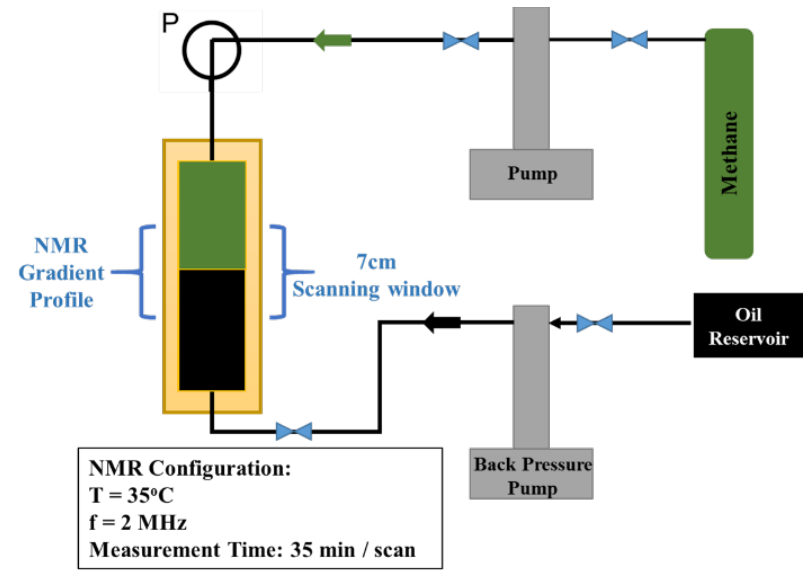

Fig. 1. Experimental setup, including NMR transparent $\mathrm{ZrO}_{2}$ pressure cell, placed within $2 \mathrm{MHz}$ NMR spectrometer. The oil phase was injected from a downstream port; the gas phase was injected through an upstream port, with the pressure controlled by a computer controlled Teledyne ISCO syringe pump system. The cell was positioned inside the spectrometer, in which the oil-gas interface was located in the middle of the gradient window.

\section{Experimental results}

With the contrast in HI between the gas and the oil phases, we can monitor the change in elevation of the interface (Fig. 2). During the diffusion process, in which pressure is maintained constant, methane molecules would diffuse into the oil phase at certain rate. This phenomenon dynamically changes the HI profile of the oil phase as a function of time. Fig. 3 shows HI profile of the oil phase from the beginning of the experiment up to 7 days. HI at any point within the oil phase decreases over time, but the reduction rate is different depend of the relative position, with respect to the oil-gas interface. Fig. 4 shows the effect of methane diffusion on HI trends at four different positions, note the marker colors correspond with the position, labeled in Fig. 3.

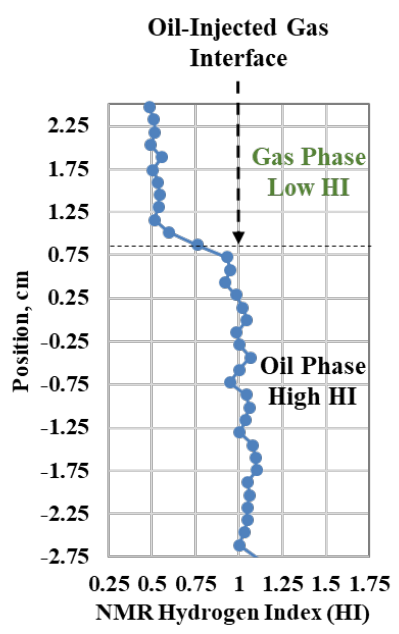

Fig. 2. An HI profile across the oil-gas interface. The position values are based on the relative position of the test cell in the NMR scanning window. The contrast in HI between the gas (low value) and the oil (high value) phases, allows to determine the dynamic position of the oil-gas interface during the experiment.

As expected, at the positions closest to the interface, HI decreased at the fastest rate, and quickly approached a constant value, $\mathrm{HI}_{\text {final. }}$. Moving further away from the interface, HI decreased with slower slope, but eventually reached the same $\mathrm{HI}_{\text {final }}$. $\mathrm{HI}_{\text {final }}$ is the result of the mixing between original oil's molecules and injection gas' molecules (methane in this case) at a particular concentration. From the experimental results, this specific concentration can be regarded as the maximum concentration of methane that can be diffused into the oil body; this parameter is a function of pressure and temperature. Using each of these HI trends, the methane diffusion coefficient can be calculated. However, with the fluctuation in the data, due to NMR signal-to-noise, the integral of HI profile is used to better estimate the diffusivity. 


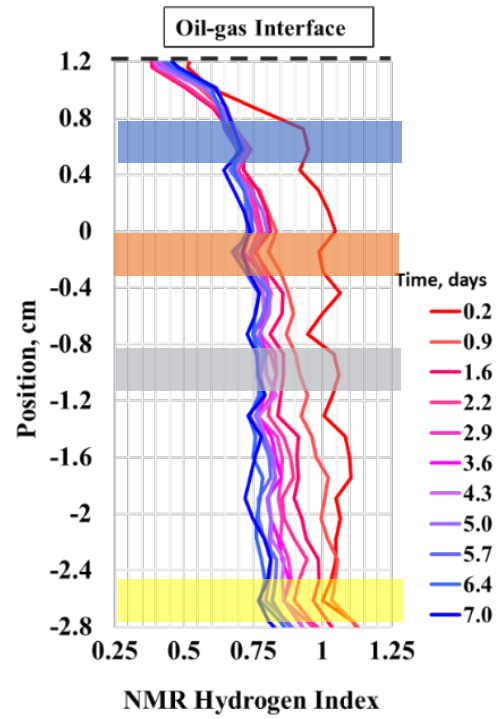

Fig. 3. HI profile within the oil phase as a function of time. The decreasing of the HI value within the oil phase is due to the methane diffusion. HI temporal profiles at different positions (color labels) within the oil phase are plotted in Fig. 4.

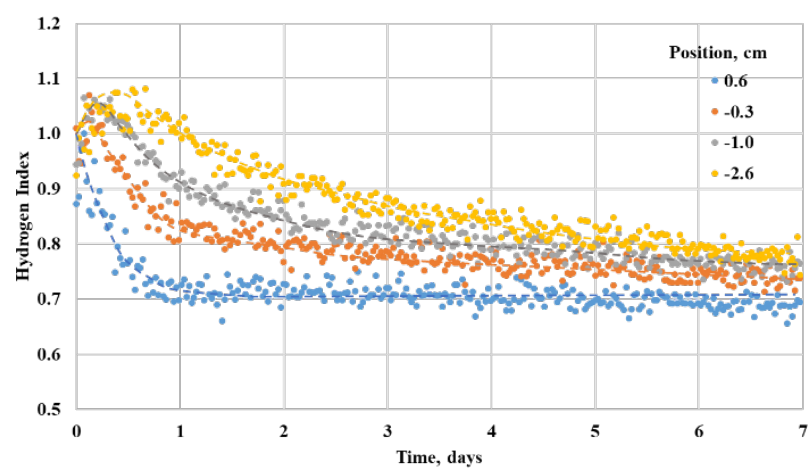

Fig. 4. HI profiles at different positions within the oil phase. The marker colors correspond to highlighted depths in Fig. 3. At the position closest to the oil-gas interface, HI decreases with the faster rate, and eventually approach a constant value, $\mathrm{HI}_{\text {final }}$. $\mathrm{HI}_{\text {final }}$ corresponds to the maximum concentration of diffused methane into the oil phase.

At a particular position and time, the HI value is the molar averaging between the HI value of the original oil and the HI value of methane at 6000 psi (Eq. 1). With the known HI value of the original oil is 1 , and known $\mathrm{HI}$ value of methane at $6000 \mathrm{psi}$ is 0.525 (both values can be extracted from the HI profile at the beginning, i.e. at $\mathrm{t}=0$ ), relative methane concentration can be calculated, then plotted as a function of time. Fig. 5 shows the integral of HI profile (red) and calculated methane concentration in the oil phase (blue.)

$$
\begin{gathered}
H I_{\text {oil phase }}(t)=(1-\alpha) H I_{\text {original oil }} \\
+\alpha H I_{\text {methane } @ 6000 p s i} \\
\alpha(t)=\frac{c_{\text {methane }}(t)}{c_{\text {methane }}(t)+c_{\text {oil }}(t)} \\
H I_{\text {original oil }}=1 \\
H I_{\text {methane } @ 6000 p s i}=0.525
\end{gathered}
$$

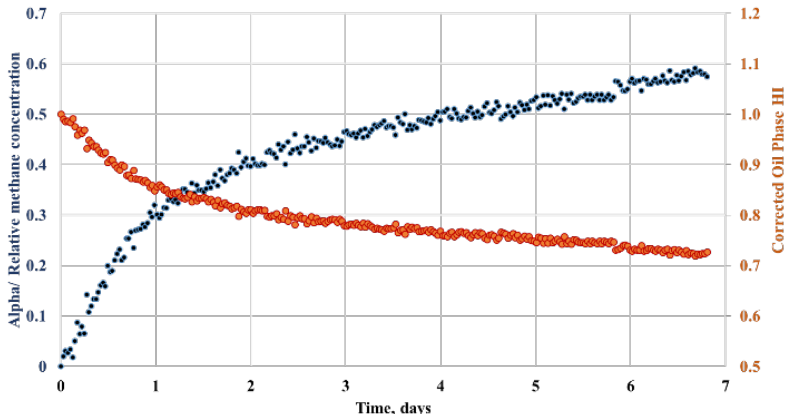

Fig. 5. The integral of HI respective to position, plotted as a function of time (red). Calculated relative methane concentration is also plotted (blue). The data is used to estimate methane diffusion coefficient.

\section{Discussions}

Fig. 4 show HI trends at different positions within the oil phase. Depending on the relative position to the interface, the HI reduction rate would be different; however, they all approach a similar value of $\mathrm{HI}_{\text {final }}$. In the other words, for a specific reservoir fluids and injection gasses, at a particular P-T condition, there is a maximum concentration of gas that can be introduced into the oil phase (Whitman, 1923[12]). While modelling the dual diffusion of injection gases into reservoir fluids and vice versa, this maximum concentration should be considered as the boundary condition; and now, we can estimate it from laboratory measurements.

Using Fick's second law (Eq. 2), diffusion coefficient can be estimated from the relative methane concentration profile. Note instead of using methane profile at a single position within the oil phase, we used the integral to reduce the impact of data oscillations. $\mathrm{C}_{\mathrm{s}}$ is methane concentration at the oil-gas interface. This is usually derived from the late-time diffusion data; however, with this study, $\mathrm{C}_{\mathrm{s}}$ can be directly calculated from $\mathrm{HI}_{\text {final }}$.

$$
\begin{gathered}
\frac{C(x, t)}{C_{s}}=1-\operatorname{erf}(z) \\
z=\frac{x}{2 \sqrt{D T}}
\end{gathered}
$$

Fig. 6 shows the fitting results of different diffusion coefficients using the relative methane concentration profile. The whole profile throughout 7 days can be fitted with diffusion coefficients ranging from $6.5 \times 10^{-10}$ to $8.5 \times 10^{-10} \mathrm{~m}^{2} / \mathrm{s}$; however, it is clear that the diffusion rate decreasing as a function of time. This is considered as experimental artifact. While Fick's Law was solved for infinite boundary condition, our test cell has limited volume. As soon as the first gas molecule travelling toward the oil phase, and approaches the end of the cell, the diffusion rate would be reduced.

By reviewing literature on diffusion measurements (Renner, 1986[13]; Grogan et al., 1988[14]; Jamialahmadi et al., 2006[11]), bulk diffusion rate can be varied within 2 orders of magnitude $\left(10^{-10}-10^{-8}\right.$ $\mathrm{m}^{2} / \mathrm{s}$ ). Translating this to field EOR applications, to 
efficiently inject a same reservoir volume, the injection time can be also varied within 2 orders of magnitude.

\section{Conclusions}

Knowing the importance of diffusion as one of major drive mechanisms in tight rocks, our studies focus on defining and estimating key parameters, allows engineers to model the process. They include porous matrix tortuosity and bulk fluid diffusivity. For example, in gas injection EOR, the combination of these two factors governs how fast injection gas molecules travel into the porous matrix to interact with a reservoir fluids' mobility, hence injection and production strategy can be optimized. In this paper, a new method to estimate fluid diffusion coefficient between methane and a crude sample was presented using NMR 1-D gradient. The technique directly captures the dynamic change of methane concentration within the oil body, reflected through the change in the HI value.

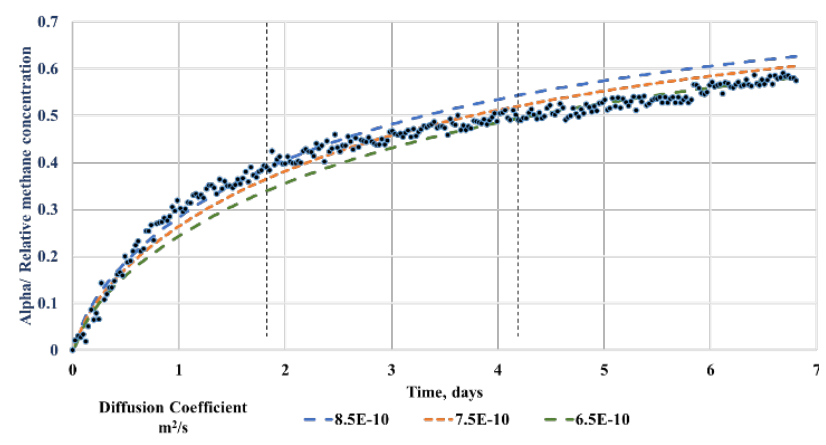

Fig. 6. The fitting of diffusion coefficient using the relative methane concentration profile. The diffusion rate can be precisely fitted within $25 \%$ of error, despite the experimental artifact, which makes diffusion rate decreases when gas molecules approach the end of the test cell.

This work was funded by the University of Oklahoma Unconventional Shale Consortium. We have benefitted from valuable conversations with Jairo Corredor, John Mansoori, Tom Smagala and Richard Newhart. The laboratory support of Gary Stowe, Micaela Langenvin and Jeremy Jernigen is greatly appreciated. We thank the members of Integrated Core Characterization Center $\left(\mathrm{IC}^{3}\right)$ for helping us during experimentation.

\section{References}

1. L. Li, Y. Su, J. J. Sheng, "Investigation of gas penetration depth during gas huff-n-puff EOR process in unconventional oil reservoirs", Society of Petroleum Engineers, 2018

2. L. Li, Y. Su, J. J. Sheng, Y. Hao, W. Wang, Y. Ly, Q. Zhao, H. Wang, "Experimental and numerical study on $\mathrm{CO}_{2}$ sweep volume during $\mathrm{CO}_{2}$ huff-n-puff enhanced oil recovery process in shale oil reservoirs", Energy Fuels, 2019

3. S. T. Dang, C. H. Sondergeld, C. S. Rai, "Effects of temperature and gas pressurization on the interpretation of nuclear magnetic resonance (NMR) hydrocarbon measurements in organic rich shales", URTeC, 2019

4. M. Cronin, H. Emami-Meybodi, R. T. Johhns, "Diffusion-dominated proxy model for solvent injection in ultra-tight oil reservoirs", Society of Petroleum Engineers, 2018

5. F. A. Coutelieris, M. E. Kainourgiakis, A. K. Stubos, "The effects of Peclet on the Sherwood number in high porosity granular media", Studies in Surface Science and Catalysis, Vol 144, p. 753-760, 2002

6. M. Fleury, E. Brosse, "Transport in tight rocks", AGU Hydrobiogeochemical Properties of Caprock, June, 2017

7. S. T. Dang, C. H. Sondergeld, C. S. Rai, A, O. Tinni, N. Drenzek, 2018, "A first step in evaluating the role of diffusion in EOR in tight shale formations", SCA, 2018

8. N. Iversen, B. B. Jorgensen, "Diffusion coefficients of sulfate and methane in marine sediments: influence of porosity", Geochimica et Cosmochimica Acta 57, 571-578, 1993

9. P. M. Sigmund, "Prediction of molecular diffusion at reservoir conditions, Part 1-measurement and prediction of binary dense gas diffusion coefficients", J. Can. Pet. Technol., 15, pp. 48-57, 1976

10. P. Gou, Z. Wang, P. Shen, J. Du, "Molecular diffusion coefficients of the multicomponent gascrude oil systems under high temperature and pressure", Ind. Eng. Chem. Res, 48, p. 9023-9027, 2009

11. M. Jamialahmadi, M. Emadi, H. Muller-Steinhagen, "Diffusion coefficients of methane in liquid hydrocarbons at high pressure and temperature", Journal of Petroleum Science and Engineering. Vol 52, 1-2, p. 47-60, 2006

12. W. G. Whitman, "The two-film theory of absorption", Chem. Metall. Eng., 29, pp. 147-152, 1923

13. T. A. Renner, "Measurement and correlation of diffusion coefficients for $\mathrm{CO}_{2}$ and rich-gas applications", Society of Petroleum Engineers, 1988

14. A. T. Grogan, V. W. Pinczewski, G. J. Ruskauff, F. M. Orr, "Diffusion of $\mathrm{CO}_{2}$ at reservoir conditions: models and measurements", Society of Petroleum Engineers, 1988 